\title{
JOSÉ ANTONIO CARO GÓMEZ: EVOLUCIÓN DE LAS INDUSTRIAS ACHELENSES EN LAS TERRAZAS FLUVIALES DEL BAJO GUADALQUIVIR (780.000-40.000 B.P.): EPISODIOS GEOMORFOLÓGICOS Y SECUENCIA PALEOLÍTICA
}

\section{EVOLUTION OF ACHEULIAN INDUSTRIES IN THE FLUVIAL TERRACES OF THE LOWER GUADALQUIVIR (780.000 - 40.000 B.P.): GEOMORPHOLOGICAL EPISODES AND PALAEOLITHIC SEQUENCE}

\author{
por
}

\author{
JOSÉ ANTONIO CARO GÓMEZ*
}

RESUMEN La estructuración de la Secuencia Paleolítica del Bajo Guadalquivir se define a través de la relación de sus industrias líticas con los depósitos aluviales, la caracterización tecnotipológica y grado de rodamiento de las mismas. En el estudio se han utilizado las industrias en conexión estratigráfica, teniendo en cuenta a su vez la variedad de formaciones sedimentarias paleoedáficas, de génesis y evolución diferentes que conforman los depósitos tipo: barras basales de gravas, bancos de arenas, lacustres, llanuras de inundación, barras laterales, horizontes de suelo y coluviones.

La posición de las industrias en la secuencia fluvial relativa y la correlación paleomagnética demuestran el desarrollo cronoestratigráfico desde el límite Pleistoceno Inferior-Pleistoceno Medio (780.000 B.P.) y el Pleistoceno Superior antiguo (hasta el 40.000 B.P.). Como conclusión se presenta una tabla de síntesis con las formaciones cuaternarias, cronologías, fauna y yacimientos paleolíticos.

ABSTRACT A final schema of the Palaeolithic sequence in the Lower Guadalquivir basin is presented. This has been achieved working ont the relationships between lithics and their bearing deposits, along with the technotypological assemblages defined, using the degree of roundiness as a taphonomic bias indicator.

Only lithics within sedimentary sections were considered. These contexts show a variety in origins and evolution that defines a number of type-deposits: gravel bars, overbank sandy deposits, lacustrine deposits, floodplain, lateral gravel bars, palaeoedaphic surfaces and colluviums.

The position of the lithic assemblages in the relative fluvial sequence and palaeomagnetic correlation revealed a chronostratigraphic complex ranging from the limit Lower-Middle Pleistocene (780.000 B.P.) till the Upper Pleistocene (40.000 B.P.). A synthetic chart shows the correlation among quaternary episodes, dates, fauna and Palaeolithic sites.

\footnotetext{
* Departamento de Geografía Física y Análisis Geográfico Regional. Universidad de Sevilla. Grupo de investigación “Cuaternario y Geomorfología”. Rnm-203. Correo electrónico: joseacarog @ terra.es
} 
Palabras claves Pleistoceno, Terrazas fluviales, Achelense, Series líticas, Rodamientos, Bajo Guadalquivir.

Key words Pleistocene, Terraces, Acheulian, Lithic series, Roundiness, Lower Guadalquivir Basin.

\section{INTRODUCCIÓN}

Los estudios arqueológicos y geomorfológicos en las formaciones aluviales del Guadalquivir han sufrido una importante evolución a lo largo del último siglo, desde los primeros trabajos de la década de los años 20 (Carandell 1925) y de los años 50 (Cabanas 1957); siendo las primeras noticias sobre la existencia de industria lítica del Paleolítico Inferior en relación con las terrazas fluviales del Guadalquivir en Carmona (Sevilla), debidas a Martínez Santa-Olalla (Sáez Martín 1956). Sin embargo, todas ellas no serían mas que meras noticias aisladas o faltas de una continuidad en la investigación que diera coherencia al estudio y a sus resultados ${ }^{1}$.

Será a finales de los años 70 y comienzo de los 80 , cuando la investigación del Paleolítico sufra un cambio cualitativo y cuantitativo para plantearse de manera sistemática, con los trabajos preliminares de prospección del territorio iniciados por E. Vallespí y G. Álvarez que en colaboración con otros investigadores darán su fruto con las primeras publicaciones (Vallespí y otros 1981; Vallespí y otros 1982).

Este planteamiento servirá de punto de partida a las investigaciones sobre las formaciones cuaternarias y Secuencia Paleolítica en el Bajo Guadalquivir, llevadas a cabo por un equipo multidisciplinar integrado por investigadores de distintos Departamentos de la Universidad de Sevilla, y coordinado por los profesores E. Vallespí (Dpto. de Prehistoria y Arqueología) y F. Díaz del Olmo (Dpto. de Geografía Física y Análisis Geográfico Regional) ${ }^{2}$.

En el desarrollo del mismo se pudo establecer una secuencia integral del cuaternario continental tanto desde un punto de vista geomorfológico como cultural. Para ello, se llevaron a cabo una serie de prospecciones superficiales y trabajos de campo que derivaron en la elaboración y conclusión de tres Tesis Doctorales (Baena 1993; Fernández Caro 1998 y Caro Gómez 1999), cuyos resultados han permitido documentar una gran cantidad de yacimientos arqueológicos en conexión con los depósitos cuaternarios del Guadalquivir y la caracterización de las formaciones morfosedimentarias ${ }^{3}$; convirtiéndose así en una de las Secuencias Fluviales Paleolítica más completa de la Península Ibérica.

\section{MATERIALES Y MÉTODO}

La sistematización de la investigación a supuesto una búsqueda de información, tanto en el ámbito arqueológico como geomorfológico, extendida a lo largo de todo el espacio geográfico del Bajo Guadalquivir (considerando como tal el tramo comprendido entre la desembocadura del Genil y las marismas) que nos permitiera el estudio, conocimiento y desarrollo de la Secuencia Cultural a partir de los materiales líticos recuperados en los depósitos de los yacimientos, su situación estratigráfica y su cronología.

1. Sobre los estudios paleolíticos desarrollados en el Valle del Guadalquivir se pueden consultar los siguientes trabajos (vid. Vallespí, E. 1992; Cortés, M. et al. 1996).

2. Estas investigaciones se han venido llevando a cabo durante el período 1985-1992, como proyecto arqueológico de la Dirección General de Bienes Culturales de la Consejería de Cultura y Medioambiente de la Junta de Andalucía.

3. Todos los trabajos han derivado en un amplio número de publicaciones, que de manera particular o dentro de valoraciones generales, han contribuido a la difusión de sus resultados. En la bibliografía se relacionan algunos de los más recientes. 


\subsection{Desarrollo cronoestratigráfico}

La cronoestratigrafía regional (bajo valle del Guadalquivir) descansa sobre los estudio multidisciplinares llevados a cabo por los investigadores de los Departamentos de Geografía Física y Análisis Geográfico Regional y de Prehistoria y Arqueología de la Universidad de Sevilla (Fig. 1), con el establecimiento de la Secuencia General del Guadalquivir (Díaz del Olmo y otros 1993), en cuya sistematización se pueden establecer tres aspectos diferenciadores pero, al mismo tiempo, complementarios:

- la secuencia relativa

— la correlación paleomagnética

— las dataciones absolutas

A las que se puede añadir de forma puntual la valoración de los restos faunísticos de las terrazas medias y bajas.

Teniendo en cuenta los derivados de esta investigación y referentes a estos aspectos la cronosecuencia aluvial y su relación con el desarrollo posterior sería:

- Altas topografías y terrazas muy altas, +169-161 m y +215 m (T1, T2, T3 y T4), serían manifestaciones anteriores a 800.000 B.P., sin evidencias de industria lítica.

- Complejo de terrazas altas (T5, T6, T7, T8 y T9) entre +139 y +73 m, con una cronología comprendida entre menos de 780.000 B.P. (límite Pleistoceno inferior-medio) y más de 300.000 B.P. Con la localización en los momentos iniciales (T5, Cerro Higoso I) de industria lítica.

- Complejo de terrazas medias (T10, T11 y T12) entre +55 y +26 m; cronológicamente están comprendidas entre 300.000 y 80.000 B.P. Con un gran desarrollo de la industria lítica.

- Complejo de terrazas bajas y muy bajas (T13) a +13-20 m, con cronología de Pleistoceno superior a Holoceno. Igualmente con presencia de industria lítica.

El desarrollo sobre estos niveles aluviales de una compleja evolución que comprende suelos, coluviones, arroyos, etc., donde se encuentra una gran parte de la industria lítica, y su correlación cronológica supone uno de los handicaps más importantes en nuestra investigación.

Para los coluviones, la cronología propuesta en razón de su posición geomorfológica, abarcaría desde el pleno Pleistoceno Medio hasta el Pleistoceno Superior antiguo, ofreciendo varias generaciones.

Estos coluviones van desmantelando las zonas más superficiales de las terrazas (suelos, llanuras de finos, etc.) lo que indica que debió pasar un tiempo suficiente desde la formación de la terraza que permitiera el abandono por parte del río y la formación de dichos niveles superficiales, que serían ocupados por los homínidos dejando allí los restos de sus actividades.

Teniendo en cuenta que la ocupación de estas partes superficiales de las terrazas (T5, T6, T7...) debió ser anterior a este desmantelamiento por parte de los coluviones, en las terrazas más altas (al menos hasta la T8) esta ocupación debe entenderse como anterior al pleno Pleistoceno Medio que es cuando comienzan a actuar los coluviones, y si estos coluviones contienen la industria mencionada ésta debe ser relacionada con una cronología no posterior a 300.000 B.P. y no anterior a 450.000-500.000 B.P., es decir, un momento inmediatamente anterior al pleno Pleistoceno Medio para las industrias de los coluviones relacionados con los niveles de las terrazas más altas.

Por otro lado, los coluviones de las terrazas medias y quizás los de la T9 deberán ser encuadrados en una cronología posterior en torno al final del Pleistoceno Medio y fases iniciales del Pleistoceno Superior correlacionable con la formación de la T12 (<200.000 B.P.), por lo que la industria contenida en ellos debe ser anterior a esta fecha. 
Por su parte en los afluentes secundarios que forman la red de drenaje de las terrazas existen, al menos, dos fases distintas de acumulación. Una más antigua representada por la primera gran discontinuidad que se produce con respecto a los depósitos aluviales de las terrazas (El Rubio, Lagunillas...), y una segunda que entraría erosivamente sobre todo el conjunto anterior.

Cronológicamente hay que tener en cuenta que los coluviones rojos más antiguos están sobreimpuestos a la primera incisión por lo que deben ser posteriores a ésta y estar relacionados con el primer gran salto topográfico en la secuencia aluvial que se produce desde la T9 hacia la T10. Por todo ello, la primera incisión de los arroyos debería ser cronológicamente asimilable a un momento inmediatamente anterior al Pleno Pleistoceno Medio cuando empieza a encajarse la red de drenaje de las terrazas altas (<450.000 B.P.) y tanto estos lugares como los interfluvios ya comentados se transforman en lugares estratégicos para el desarrollo de la vida humana.

Con posterioridad y en relación con el $2^{\circ}$ gran salto topográfico, el de la T12 sobre la T13, podría situarse la $2^{\mathrm{a}}$ fase de acumulación que se desarrollaría a finales del Pleistoceno Medio y durante el Pleistoceno Superior antiguo (200.000-40.000 B.P.); acumulando en sus depósitos las industrias recogidas del desmantelamiento de la fase anterior y las propias de su época, con la circunstancia que en los niveles superiores de estos arroyos se encuentra bien situada estratigráficamente una industria característica del Paleolítico Medio, por lo que parece consecuente no llevar su formación más allá de las fechas indicadas ${ }^{4}$.

El desarrollo de los niveles hidromorfos, tierras negras, coluviones negros, etc., queda bien encuadrado en esta franja final establecida pues suelen cerrar las secuencias estratigráficas de gran parte de los yacimientos y formaciones estudiados, situándose a techo de las mismas y conteniendo en su interior una industria muy bien caracterizada de Paleolítico Medio. La correlación paleomagnética ha establecido para el nivel 3.2 de Saltillo la identificación de un evento negativo (Biwa 1, aprox. 180.000 B.P.), sobre el que se instala uno de estos depósitos pardo-negruzco, que por lo tanto deben poseer una cronología posterior (<100.000 B.P.).

La información que nos proporciona la fauna recuperada en la T12 -nivel Jarillas-(Elephas antiquus, Hippopotamus amphibius, Cervidae cf. Dama, Gran Bovidae indet., Lagomorpha: Leporidae indet, Equus $s p$.) a este respecto es indicativa de los períodos propios del Pleistoceno Medio y Superior del Mediterráneo Occidental $^{5}$. Climáticamente, indican un ambiente templado o algo más caluroso que en la actualidad (sobre todo, hipopótamo y elefante). La presencia de estas dos especies conlleva la existencia de agua abundante, especialmente el hipopótamo y, obviamente, una vegetación suficiente que les proporcione el abundante forraje que necesitan; datos que están en plena consonancia con el tipo de medio físico descrito más abajo y que sería el que constituyó el principal hábitat humano del Paleolítico en el valle del Guadalquivir.

\subsection{Las terrazas fluviales y la distribución espacial de las series líticas}

Los sistemas fluviales han sido unos de los objetos de investigación más trabajados y desde hace más tiempo. Es un tema tradicional en la investigación cuaternarista incluyendo los estudios paleolíticos y arqueológicos en general, pues se muestran muy ricos en aportar información paleogeográfica y yacimientos arqueológicos.

El elemento de estudio de los sistemas fluviales paleogeográficamente son las terrazas fluviales: morfologías en forma de plataformas construidas a partir de depósitos aluviales heredados que se disponen lateral

4. Es interesante reflejar aquí la situación que ofrecen otras regiones extrapeninsulares como la Dordoña (Francia) donde, al final del Pleistoceno Medio comienzos del Superior, se produce una circunstancia por la que los valles sufren un encajonamiento importante de la red hidrográfica de 20 a 40 m creando así un nivel marcado en el paisaje (Turq 1992).

5. Sólo se han podido recuperar restos paleontológicos en la T12 y T13, con una caracterización prácticamente idéntica. El estudio ha sido realizado por E. Soto. 
y perpendicularmente al sentido del curso fluvial. Cuando las terrazas conservan un modelo típico, morfológicamente se representan por una superficie relativamente horizontal que en su borde externo (hacia el río) queda limitada por una escarpada pendiente descendiente y en la otra por una nueva pendiente ascendente (Fig. 2, 1a).

Es conveniente distinguir la terraza morfológica del depósito de dicha terraza que se correspondería con el aluvionamiento, ya que la terraza podría estar retocada por diferentes motivos, sin embargo, el aluvión permanecería y a partir de él obtenemos los datos fundamentales de la cronología y el ambiente paleogenerador del mismo.

En este proceso de formación de la terraza la disminución de la velocidad de la corriente supone el abandono de una parte de la carga (el río aluviona); primero se depositan los cantos más grandes, luego las gravillas, más tarde las arenas y, finalmente, las arcillas (Fig. 2,1b). En el curso de su historia, cuando un río reactiva su potencia erosiva (por cualquier causa que aumente su caudal y la velocidad de sus aguas), ahonda su lecho en sus propios aluviones pudiendo alcanzar o no el sustrato geológico; la repetición de este fenómeno en el transcurso del Pleistoceno ha dado lugar a la formación de las terrazas escalonadas y embutidas del Guadalquivir.

La interpretación pues de la terraza a la hora de valorar los restos líticos y arqueológicos en general encontrados en cada una de ellas debe incluir dos aspectos, por un lado el estratigráfico y por otro el morfológico.

En el primero, tendremos que tener en cuenta el principio de superposición de estratos (los inferiores son anteriores a los superiores) ya que no es una unidad global sino el resultado de una serie de procesos deposicionales sucesivos en el tiempo, es decir, que estamos ante una cronología vertical (Fig. 2, 1c); por otro lado, al estudiar el conjunto formado por todas las terrazas de un valle fluvial hay que tomar en cuenta el aspecto morfológico de la evolución y disposición de todas ellas en el plano teórico horizontal, donde por el contrario a lo que sucedía en la estratigrafía hallaremos como lo que está más bajo (cerca del río) es lo más reciente, y lo que está más arriba son los niveles más antiguos (Fig. 2, 1d). O sea, que en la interpretación cronosedimentaria de las terrazas y los aluvionamientos se imbrican dos sistemas de cronología relativa, el geológico de las estratigrafías de los aluvionamientos y el morfológico de su posición geomorfológica (Fig. 2, 1e).

A la vista de esta distribución teórica se puede entender que si se desmantela parte de los aluvionamientos superiores de un nivel de terraza, la morfología resultante de ésta y su depósito aflorante podrían quedar así vinculados morfotopográficamente con otro nivel de cronología muy posterior (por ejemplo: tiempo 1 -t1- con tiempo 4 -t4-, tiempo 5 -t5- con tiempo 8 -t8-., etc.) (Fig. 2, 1f).

Esta breve reflexión no es baladí pues trabajo que presentamos realizado sobre las formaciones superficiales correlativas del Valle del Guadalquivir se presenta realmente complicado, no sólo en su estudio sino en su explicación y compresión, al tener que conjugar las diversas situaciones estratigráficas y morfogenéticas de los diferentes yacimientos líticos y establecer una cronología secuencial de los mismos, y queremos así dejar bien claro cual ha sido el proceso de interpretación seguido en el desarrollo de la investigación.

\subsection{Relación entre los depósitos aluviales y la industria lítica: indicadores de ocupación}

Por tanto, en nuestro estudio de los yacimientos paleolíticos del Bajo Guadalquivir que forman parte de su Secuencia fluvial integral, nos hemos encontrado con una importante variedad de tipos de formaciones cuaternarias, de génesis diferentes y evolución en el tiempo, en conexión con las cuales se localizaron los restos de industrias líticas. Cada uno de ellos adquiere una significación especial en virtud del proceso de formación que han debido tener los distintos yacimientos, elemento esencial para su comprensión e interpretación (Fig. 2, 2 y 3). En el análisis de esta relación entre depósitos aluviales e industria lítica se aplican dos 
indicadores; por un lado, el grado de rodamiento de las piezas, y por otro, el número de elementos recuperados en cada depósito, que nos informan sobre la ocupación de los mismos por parte de los grupos humanos.

Los datos utilizados se exponen en la tabla I (Fig. 3) donde se refleja la relación entre los diferentes niveles de terraza, las formaciones cuaternarias del Pleistoceno correspondientes y la representatividad de las series industriales recuperadas en cada una de ellas (para este estudio se dejan fuera las series de superficie).

Según estos datos vemos como los depósitos propiamente aluviales de fondo de canal, barras masivas de gravas, etc., suponen un $18 \%$ de la industria encontrada que como es lógico y consecuente con el funcionamiento de las mismas, por ser depósitos de mucha tracción, se encuentra muy afectada por el rodamiento mayoritariamente fuerte (R3), con frecuencia mediano (R2) y excepcionalmente leve o sin rodamiento (R1 y R0); cuya procedencia debe ser indudablemente asignada a dicho nivel de terraza o en todo caso a un momento anterior a su sedimentación ${ }^{6}$.

Los niveles de arenas, bien finas formando la llanura de inundación inmediata o gruesas por provenir del lavado lateral de las barras de gravas, no presentan muchos restos líticos $(0,5 \%)$, si bien es normal que debido al gran desmantelamiento que han sufrido este tipo de depósitos en las terrazas altas y medias hace que sean muy escasos en estos niveles; sin embargo, en la T12 son relativamente abundantes pero sin que la industria sea representativa, algunos de estos lugares en el límite con los depósitos limosos tienen una industria muy fresca.

Hay una serie de depósitos lacustres, vinculados a las terrazas altas, formados por carbonatos arenoarcillosos en cuyo interior se suelen encontrar flotando gravas e industria lítica que suponen un bajo porcentaje $(0,4 \%)$, el rodamiento afecta a esta industria de manera variada.

Las localizaciones de industria lítica en los depósitos de llanuras aluviales que genéricamente incluirían niveles de limos, arcillas, con desarrollo posterior o no de suelos rojos, zonas de encharcamientos que evolucionan a tierras negras, etc. (depósitos de finos a techo de los sistemas propiamente aluviales), son muy abundantes con una representatividad del $28 \%$; la industria aparece en estos lugares mayoritariamente fresca o con un leve redondeamiento de sus aristas más en función de un "accionamiento acuoso" (Santonja, M. y Pérez González 1984), acción eólica, etc. que a un propio rodamiento fluvial, puesto que las características de los depósitos apenas permiten el desplazamiento de la misma, por lo que nos encontraríamos ante lugares primarios de ocupación antrópica.

En la actualidad se acepta que en el medio fluvial pueden aparecer conjuntos arqueológicos en múltiples condiciones de conservación, incluyendo sitios en posición primaria y otros en posición derivada, tan válidos para interpretar la naturaleza de las ocupaciones, los procesos de formación de agregado arqueológico y las modificaciones post-deposicionales como los yacimientos en cueva, a veces incluso en mejores condiciones para distinguir suelos de ocupación en sentido estricto (Moure y Santonja 1991). El desarrollo de estas formaciones se localiza a lo largo de todos los aluvionamientos de las terrazas, excepto las dos primeras más altas (T5 y T6) y la T9.

Por otro lado, las formaciones de carácter coluvial contienen en sus depósitos otro alto porcentaje de la industria lítica recuperada (17\%) con un amplio desarrollo sobre las terrazas altas (T5 hasta T10), pero con una concentración más elevada en la T7 y T9, situación lógica pues son estas dos terrazas las más extensas de estos niveles altos. La industria en ellos recogida se encuentra afectada normalmente por un rodamiento leve (R1) o moderado (R2), aunque hay ejemplos donde la mayoría de la serie está exenta del mismo; el rodamiento fuerte aparece de forma minoritaria en todos los yacimientos y localizaciones.

6. Esta circunstancia de encontrar industria lítica sin rodamiento en depósitos de alta energía, aunque no es frecuente tampoco debe de extrañarnos, pues pueden darse situaciones concretas que lo favorezcan, y que se han reconocido en otros ámbitos fluviales peninsulares: "Es evidente que en cualquier medio dinámico pueden existir cantos que en condiciones muy concretas no experimenten ningún desgaste superficial directamente observable, bien al haber sido recubiertos por material fino protector-arcilla, limo o arenao porque una posición favorable en el fondo del canal hubiera impedido cualquier desplazamiento sensible, aunque en este caso es fácil que la acción erosiva del agua produjera alguna alteración reconocible posteriormente" (Santonja 1986: 37). 
Finalmente, es en los depósitos de los afluentes secundarios (arroyos) donde encontramos el porcentaje más elevado de industria lítica en conexión (36\%); su desarrollo a lo largo de todos los niveles de terrazas hace que sea más fácil la localización de yacimientos en los mismos; de todas formas la concentración más elevada de localizaciones e industria se da en los arroyos que disectan las terrazas T7 y T8. La industria recogida presenta unas características físicas muy parecidas a la de los coluviones con una estructura en cada yacimiento donde el rodamiento leve (R1) y mediano (R2) suponen los porcentajes más representativos, aunque a veces, hay series donde el R0 obtiene una representatividad mayor, el R3 sigue siendo bajo e inexistente en algunos ${ }^{7}$.

\section{DESARROLLO DE LAS SERIES LÍTICAS PLEISTOCENAS Y ESTABLECIMIENTO DE LA SECUENCIA PALEOLÍTICA DEL BAJO GUADALQUIVIR}

Una vez analizadas las variables técnicas, tipológicas, de explotación de recursos, etc., de las series líticas y su contextualización estratigráfica, planteamos una caracterización concreta de cada uno de los momentos culturales que creemos se pueden distinguir en función de las mismas; diferenciando claramente las industrias que se desarrollan durante todo el Pleistoceno Medio entre aquellas otras que desde estos momentos finales van a desembocar en el Pleistoceno Superior antiguo. Pero además de estas grandes líneas interpretativas los conjuntos estudiados nos dan juego para plantear una caracterización más precisa sobre todo durante ese primer momento que ocupa una banda cronológica tan amplia (<700.000-120.000 B.P.) y que se corresponde con el desarrollo del Paleolítico Inferior-Achelense del Bajo Guadalquivir.

\subsection{Caracterización y atribución tecno-cultural de las series}

En el comienzo de la Secuencia Cultura del Bajo Guadalquivir existe una industria ubicada en las terrazas más altas (T5 y T6), en sus niveles aluviales y lacustre, que no presenta ningunas características que nos puedan informar sobre una posible atribución Achelense, pues sólo se compone de núcleos, productos de talla y cantos tallados en una cantidad considerable que en principio podría trasladarla a un momento preachelense, con lo cual no estamos de acuerdo, en principio.

Sin embargo, sobre la T6 hay un yacimiento situado en un coluvión (Huerta de la Reina) que tiene una industria (Fig. 4: 1 y 2) muy similar dominada en los utensilios por los cantos tallados unifaciales, pero con un hendedor, tres triedros y un bifaz fuera de contexto que por su matriz y rodamiento podría ser atribuido a este nivel, cuya conexión con dichos niveles de terraza nos parece evidente, y que nos está indicando una etapa ya Achelense; idea con la cual estamos más de acuerdo ${ }^{8}$.

En los niveles aluviales de la T7, T8 la industria está claramente relacionada con la anterior, sobre todo la T7 donde la tipología es escasa y muy uniforme, pero en la T8 el número de resto aumenta sensiblemente en algunas localizaciones y se produce un claro aumento del grupo macrolítico con la presencia ya de bifaces que dominan sobre los hendedores, también presentes; por lo tanto debemos identificar este periodo con un claro Achelense aún poco desarrollado.

7. Esta misma situación es puesta de manifiesto por otros autores: Moure Romanillo y Santonja Gómez (1991) observan como en el ámbito local se aprecia frecuentemente una preferencia acusada, por parte de los grupos humanos, por ocupar la red fluvial secundaria, especialmente por zonas de confluencia con el valle principal.

8. Son diversas las ocasiones en que se han relacionado los primeros momentos achelenses con una baja incidencia de los bifaces (Villa 1983). 
Será a partir de la T9 cuando la industria se va generalizando, adquiriendo unas características más significativas como el aumento del utillaje bifacial con una mayor elaboración técnica y cierta estandarización en los utensilios sobre lasca, diversificación de tipos, etc.; culminando en los niveles de arroyos y coluviones correlacionables con este nivel y con el posterior de la T10, momento en el cual se desarrolla el gran Achelense de la región (Fig. 4: 3,4,5), evidenciado en el aumento de los yacimientos y series líticas, que ofrecen un crecimiento efectivo de los índices bifaciales que llegan a ser en ocasiones mayoritarios, una diversificación de los tipos, técnicas de talla más depuradas (Fig. 5), percibiéndose un leve cambio hacia la utilización cada vez mayor del sílex, etc.

Este proceso evolutivo irá cambiando nuevamente a partir de la T11 donde, aún siguiendo con esas características generales, difícilmente separables de las anteriores, comienza una sensible caída del macroutillaje donde ya son los hendedores los que dominan, aumentan los índices levallois, los utensilios del grupo Paleolítico Superior, la utilización del sílex, etc.; si bien es verdad que no de manera general sino más bien puntual en algunas series mientras que otras se mantendrán invariables y, por contra, en algunas el cambio será aún mayor; situación que ya será más patente en el próximo nivel.

Ciertamente en el nivel T12 y los yacimientos correlacionables con él, existe una yuxtaposición de situaciones pues junto con niveles cuya industria es perfectamente equiparable al momento anterior de la T11 (La Pastora, Alcolea), hay otros donde ya se notan las características propias de los momentos postachelenses.

Entre ellas es de destacar la utilización más frecuente del sílex, de manera general, como materia prima en detrimento de la cuarcita, que en algunos yacimientos sufrirá una reducción muy considerable. Sin embargo, de forma particular, encontramos yacimientos donde es la cuarcita la materia casi exclusiva empleada en la elaboración de la industria lítica (Fig. 6).

Pero esta situación hay que relacionarla también con el gran desarrollo estratigráfico de este nivel de terrazas donde la potencia llega a 15-16 m en algunos lugares, por lo que la diferencia cronológica junto al momento de transición que se parece estar viviendo nos ofrece conjuntos líticos bien diferenciados en un mismo perfil: si en el nivel inferior de Pionner (PIO-1) la industria se asemeja más a la de Tarazona I, la industria de Pionner 4 (PIO-4) es claramente de Paleolítico Medio, además con una gradación que se puede ir siguiendo en los niveles intermedios (PIO-2 y PIO-3). Por ello sería lógico que en un momento de cambios significativos la evolución no sea unilineal sino más bien multivariable con influencias locales, tradicionales o incluso foráneas, es decir, que mientras en unos lugares se está dando una adaptación a los nuevos modos técnicos en otros se siguen utilizando las viejas técnicas influenciadas aún por la tradición cultural, que en muchas ocasiones creemos no llegarán a cambiar.

Finalmente, en los niveles más superficiales de esta T12, en otros similares de terrazas anteriores, en yacimientos en superficie, etc., el cambio se hace patente con el desarrollo total de estas nuevas técnicas en unas industrias terminales del proceso de evolución seguido desde los primeros momentos achelenses (Fig. 7), cuyas características nos hacen pensar en su separación del Paleolítico Medio clásico (musteriense) para incluirlo en una fase concluyente de este proceso, como un continuum que, aún significando un impacto efectivo de nuevos modos culturales, no abandona las formas tradicionales expresadas en parte de las características de sus industrias, convirtiéndose así en un Paleolítico Medio peculiar de los ámbitos fluviales peninsulares.

\subsection{Estructuración de la secuencia paleolítica}

Tras estas consideraciones presentamos una Secuencia Cultural Paleolítica (Fig. 8) en las terrazas fluviales del Bajo Guadalquivir que quedaría vertebrada con relación a las siguientes fases evolutivas:

1.- En primer lugar tenemos unas industrias escasas, sin una caracterización precisa, en los niveles aluviales de terrazas altas T5 y T6 $(+139 \mathrm{a}+110 \mathrm{~m})$, Cerro Higoso I y II, que deben ser consideradas, en principio, 
como un Paleolítico Inferior de carácter indeterminado, pero que creemos forma parte ya de la secuencia Achelense en su fase inicial (indicando un posible Achelense antiguo), sobre la base del yacimiento coluvionar de la fase antigua de Huerta de la Reina, situado en una posición cronoestratigráfica de la T6 (Cerro Higoso II).

2.- El complejo de terrazas altas, representado en los niveles T7 a T9 (+100 a +73 m), junto con los dos primeros de las terrazas medias T10 y T11 $(+55 \mathrm{a}+35 \mathrm{~m})$ los coluviones y arroyos correspondientes deben interpretarse como el momento del desarrollo general del Achelense en el bajo Guadalquivir, con una inseparable evolución influenciada por la tradición cultural las industrias en valles fluviales, pero a lo largo de la cual se pueden matizar varios momentos indicativos de la variabilidad lógica inherente a toda evolución cultural.

En un primer momento, relacionado con los depósitos aluviales de la T7, T8 (Muharra-1, Toril-1, Monclova, Usea-1, etc.) y algunos coluviones y fases iniciales de arroyos (Mingalario-1, Sargenta, etc.), estaría la implantación efectiva de este Achelense (Achelense en Terrazas Altas), que hacia la T9 (Tambor, Gastaembalde, etc.) continuando sobre la T10, T11 (Harinera-1, Tarazona I, Guadajoz, etc.) y todo el desarrollo coluvio-aluvial contemporáneo a las mismas (La Celada, El Ciervo, Santiche, etc.), supone la gran explosión del poblamiento Achelense y el desarrollo de sus características tecno-culturales (Achelense en Terrazas Medias-Altas); para finalmente y a partir ya de la T11 (Espartales, El Caudal, etc.) y sobre todo en la T12 producirse una fase de transición (Achelense Final Transicional) bien caracterizada, de yacimientos como Las Jarillas, Antiguos Viveros, Pionner-1, MH4, que evidencian ya unas características evolutivas más avanzadas cercanas al Paleolítico Medio, pero que como hemos visto no suponen un compartimento cerrado, sino que su propio carácter de transición, hace que nos encontremos con conjuntos que de manera general manifiestan una línea de evolución parecida, pero que particularmente se ve reflejada en elementos diferentes en cada uno de los conjuntos ${ }^{9}$.

De tal forma, este cambio que se aprecia desde finales del Pleistoceno Medio no se presenta en forma de ruptura sino de transformación, desde unas industrias con una marcada tradición Achelense hasta otras que pueden desembocar en un nuevo modo técnico, adoptando las modernas tradiciones, aunque sin perder de vista las anteriores (Pionner 2 y 3 ), o permanecer invariables prácticamente hasta el final de la Secuencia establecida (La Pastora-1, Alcolea-1); o incluso mezclarse de tal manera que es altamente difícil atribuir alguna serie (Tarazona II) a una u otra tradición cultural de forma concluyente.

3.- Todo el proceso seguido desembocará en unas industrias que forman el Paleolítico Medio regional, donde los caracteres evolutivos de naturaleza técnica, tipológica y de comportamiento en general, lo separan claramente tanto del Achelense como del Musteriense clásico, confiriéndoles una personalidad propia (Saltillo-2, El Caballo, Aeropuerto-2, Pionner-4, etc.) (Paleolítico Medio de ámbitos fluviales) ${ }^{10}$.

En conclusión, esta secuencia cultural refleja una homogeneidad tecno-morfológica que aparece expresada en una continuidad cultural, relacionada con los complejos de cantos tallados de las depresiones fluviales, sólo interrumpida por paulatinos impactos sucesivos de nuevas tecnologías que no desprecian las anteriores, más bien las complementan, quedando siempre un substrato arcaizante de cantos tallados, grupo macrolítico, etc., que se prolonga incluso hasta el Paleolítico Medio, configurándose así una Secuencia Paleolítica regional inseparable que abarcaría, al menos, todo el Pleistoceno Medio y el Pleistoceno Superior antiguo.

9. En Francia parece desarrollarse una situación parecida: "El achelense final de las zonas septentrionales de Francia muestra unos rasgos evolutivos muy específicos, con industrias tipológicamente mucho más variadas que en momentos anteriores y que está en la base del Paleolítico medio. Los caracteres más sobresalientes son la disminución o ausencia de bifaces, el aumento y diversificación tipológica del utillaje sobre lasca, especialmente de los grupos I y III de Bordes" (Tuffreau 1979, 1981).

10. Paleolítico medio de aspecto postachelense para E. Vallespí $(1992,1994)$. 


\section{BIBLIOGRAFÍA}

BAENA ESCUDERO, R. (1993): "Evolución Cuaternaria (3 M.a.) de la Depresión del Medio-Bajo Guadalquivir y sus Márgenes (Córdoba y Sevilla)". Tesis Doctoral. Facultad de Geografía e Historia de Sevilla. Inédita.

BAENA, R. y DÍAZ DEL OLMO, F. (1994): "Cuaternario aluvial de la depresión del Guadalquivir: episodios geomorfológicos y cronología paleomagnética", Geogaceta 15: 102-103.

BAENA, R. y CARO, J.A. (1993): "Secuencia aluvial y postaluvial de la terraza de Muharra (Carmona, Sevilla). Aportación al Achelense en el valle del Guadalquivir", Spal 3: 51-71.

BAENA, R. y DÍAZ DEL OLMO, F. (1997): "Estratigrafía compleja en terrazas fluviales del Guadalquivir: formaciones aluviales y paleomagnetismo en Cerro Higoso (Carmona, Sevilla)", en Rodríguez Vidal, J. (ed.). Cuaternario Ibérico: 317-321. Huelva.

CABANAS, R. (1957): "Las terrazas cuaternarias del Guadalquivir y sus afluentes en la provincia de Jaén", Rev. R. Acad. Cien. Ex. Fís. y Nat. de Madrid, 51, $2^{\circ}$ y $3^{\circ}: 147$.

CARANDELL, J. (1925): "Las terrazas cuaternarias del río Guadalquivir", Ibérica 24 (604): 328-331.

CARO GÓMEZ, J.A. (1999): "Conjuntos achelenses de la Terrazas Altas y Medias del Bajo Guadalquivir: yacimientos de Muharra, Arroyo El Rubio, Tarazona I y El Caudal”, en Avances en el estudio del Cuaternario Español. (Secuencias, indicadores paleoambientales y evolución de procesos). Eds.: L. Pallí Buxó y C. Roqué Pau: 241-246. Servei de publications Universitat de Girona.

- (1999): "Yacimientos e industrias achelenses en las terrazas fluviales de la depresión del Bajo Guadalquivir (Andalucía, España). Secuencia estratigráfica, caracterización tecnocultural y cronología". Tesis Doctoral (inédita). Universidad de Sevilla.

CORTES, M.; MUÑOZ, V.E.; SANCHIDRIÁN, J.L. y SIMÓN, M.D. (1996): El Paleolítico en Andalucía. Córdoba.

DÍAZ DEL OLMO, F.; VALLESPÍ, E. y BAENA, R. (1993): “Cuaternario y secuencia paleolítica en las terrazas del Bajo y Medio Guadalquivir: aluvionamientos, coluviones, suelos y paleosuelos", Investigaciones arqueológicas en Andalucía, 1985-1992. Proyectos VI. Jornadas de Arqueología Andaluza. Huelva.

DÍAZ DEL OLMO, F. y BAENA, R. (1997): "Interpretación de la Secuencia General del Guadalquivir (Valle Medio y Bajo): Terrazas fluviales y Paleolítico", Cuaternario Ibérico, AEQUA. Edt. J. Rodríguez Vidal: 273-282. Huelva.

FERNÁNDEZ CARO, J.J. (1998): Las industrias líticas paleolíticas del Bajo Guadalquivir: Río Corbones. Tesis Doctoral inédita. Universidad de Córdoba.

FERNÁNDEZ CARO, J.J.; BAENA ESCUDERO, R. y GUERRERO AMADOR, I. (1999): "Poblamiento e industrias líticas a orillas del río Corbones (La Puebla de Cazalla, Sevilla)", 125 pgs. Cuadernos de Historia. Ayuntamiento de La Puebla de Cazalla.

MOURE ROMANILLO, A. y SANTONJA GÓMEZ, M. (1991): "La renovación de los estudios sobre Paleolítico en los últimos veinte años", Boletín de la Asociación Española de Amigos de la Arqueología 30-31: 13-25.

SÁEZ MARTÍN, B. (1956): Noticiario Arqueológico Hispánico III y IV. Cuadernos 1-3(1954-55): 219-223 y 232.

SANTONJA, M. (1986): "Valgrande (Puebla de Yeltes, Salamanca): área de talla y sitio de ocupación del Paleolítico Medio", Numantia. Investigaciones Arqueológicas en Castilla y León II: 33-85.

SANTONJA, M. y PÉREZ GONZÁLEZ, A. (1984): "Las industrias paleolíticas de La Maya I en su ámbito regional", E.A.E. 135. Ministerio de Cultura. Madrid. 
TUFFREAU, A. (1979): "Les débuts du Paléolithique moyen dans la France septentrionale", Bull. Soc. Preh. Franç. París 76: 140-142.

(1981): "L'Acheuléen dans la France septentrionale", Anthropologie XIX, 2: 171-183. París.

TURQ, A. (1992): "Le Paléolitique Inférieur et Moyen entre les Vallées de la Dordogne et du Lot", These, ejemplar policopiado.

VALLESPÍ, E. (1992): “Las industrias achelenses en Andalucía: ordenación y comentarios”, Spal 1: 61-78. Universidad de Sevilla.

(1994): "El Bajo Guadalquivir en el Paleolítico Inferior y Medio peninsular", en Homenaje al Dr. Joaquín González Echegaray. Museo y Centro de Investigación de Altamira. Monografías no 17: 13-16. (2000): "Comentario al Paleolítico Inferior Ibérico: continuidad, etapas y perduraciones del proceso tecnocultural", Spal 8: 39-46. Universidad de Sevilla.

VALLESPÍ, E.; AMO, M. y ÁLVAREZ, G. (1981): "Primeras evidencias paleolíticas en la provincia de Huelva", Huelva Arqueológica V: 9-29.

VALLESPÍ, E.; ÁLVAREZ, G.; AMORES, F. y ESCACENA, J.M. (1982): "Complejo de cantos tallados y bifaces en el Bajo Guadalquivir: perspectivas de su estudio", Grupo Español de Trabajo del Cuaternario, $5^{a}$ Reunión, Conferencias: 79-94. Universidad de Sevilla.

VALLESPÍ, E. y DÍAZ DEL OLMO, F. (1996): "Industries in quarzite and the beginning of the use of flint in the Lower and Middle Palaeolithic Sequence of the Bajo Guadalquivir", Non-Flint Stone tooles and the Palaeolithic Occupation of the Iberian Peninsula, Ed. N. Moloney; L. Raposo; M. Santonja, BAR International Series 649: 135-140. Oxford.

VILLA, P. (1983): Terra Amata and the Middle Pleistocene Archeological Record of Southern France. Univ. of California Press, Berkeley. 


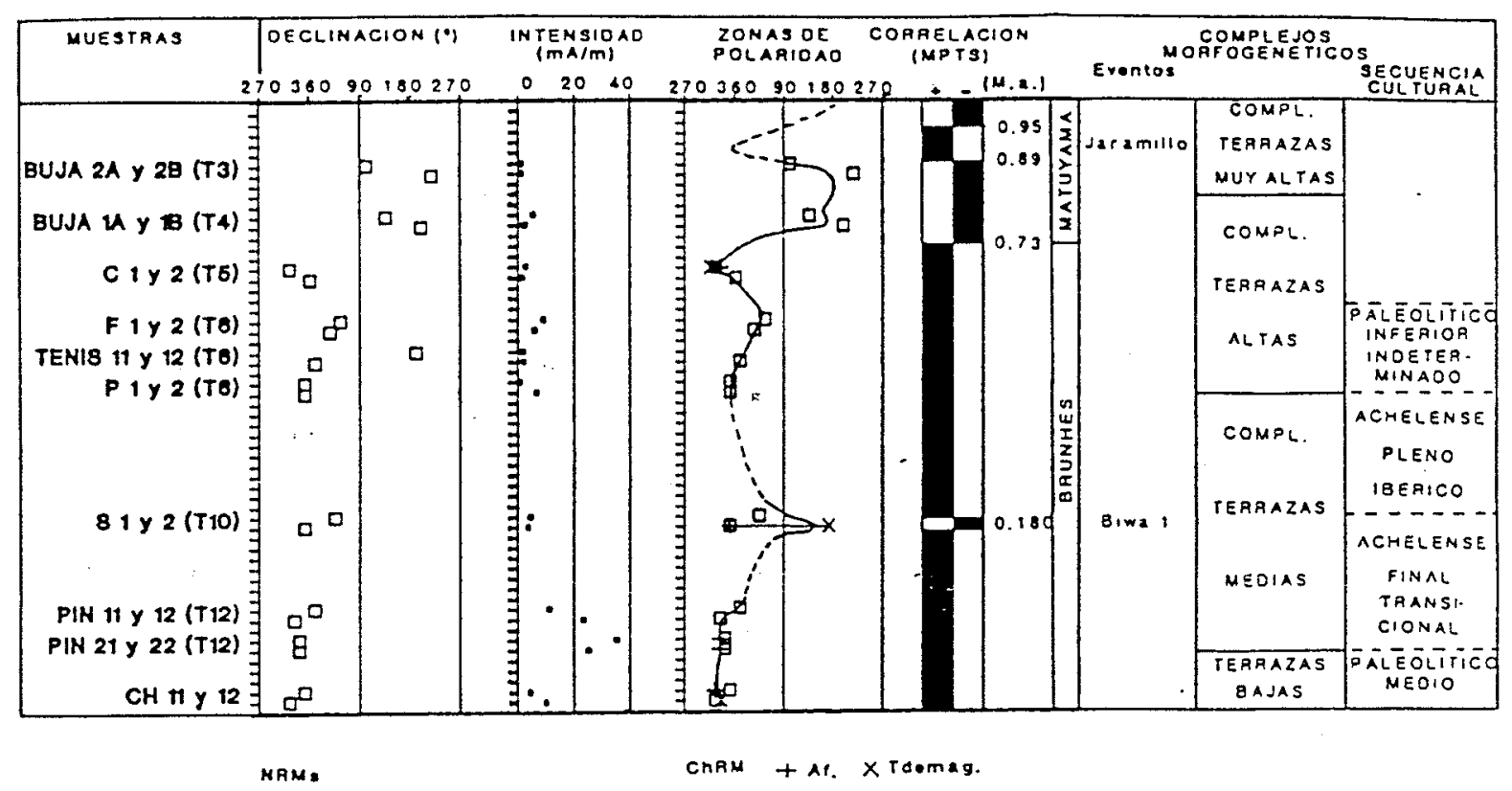

Fig. 1: Cronosecuencia de las terrazas fluviales del Guadalquivir: paleolítico, episodios geomorfológicos y paleomagnetismo (según Baena y Díaz del Olmo, 1994). 

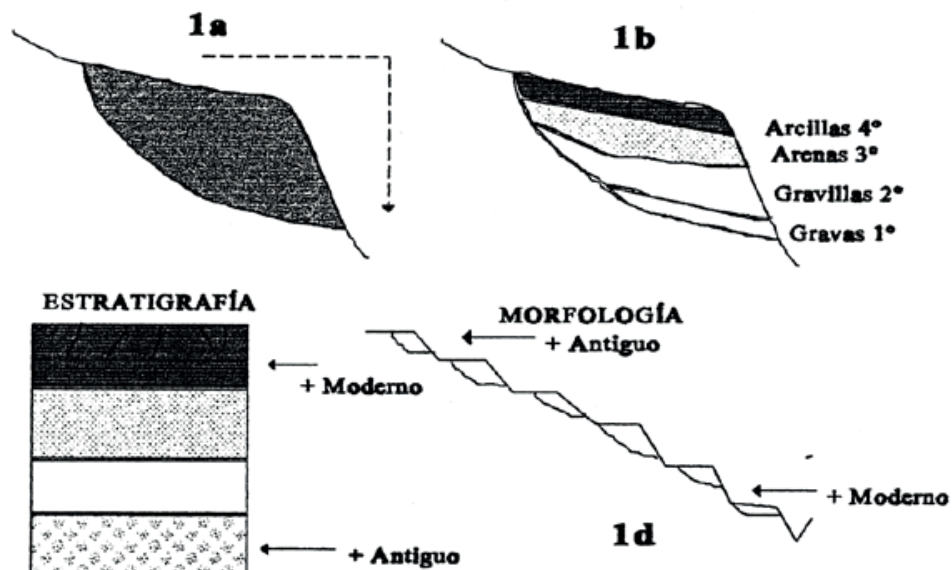

$1 \mathbf{c}$
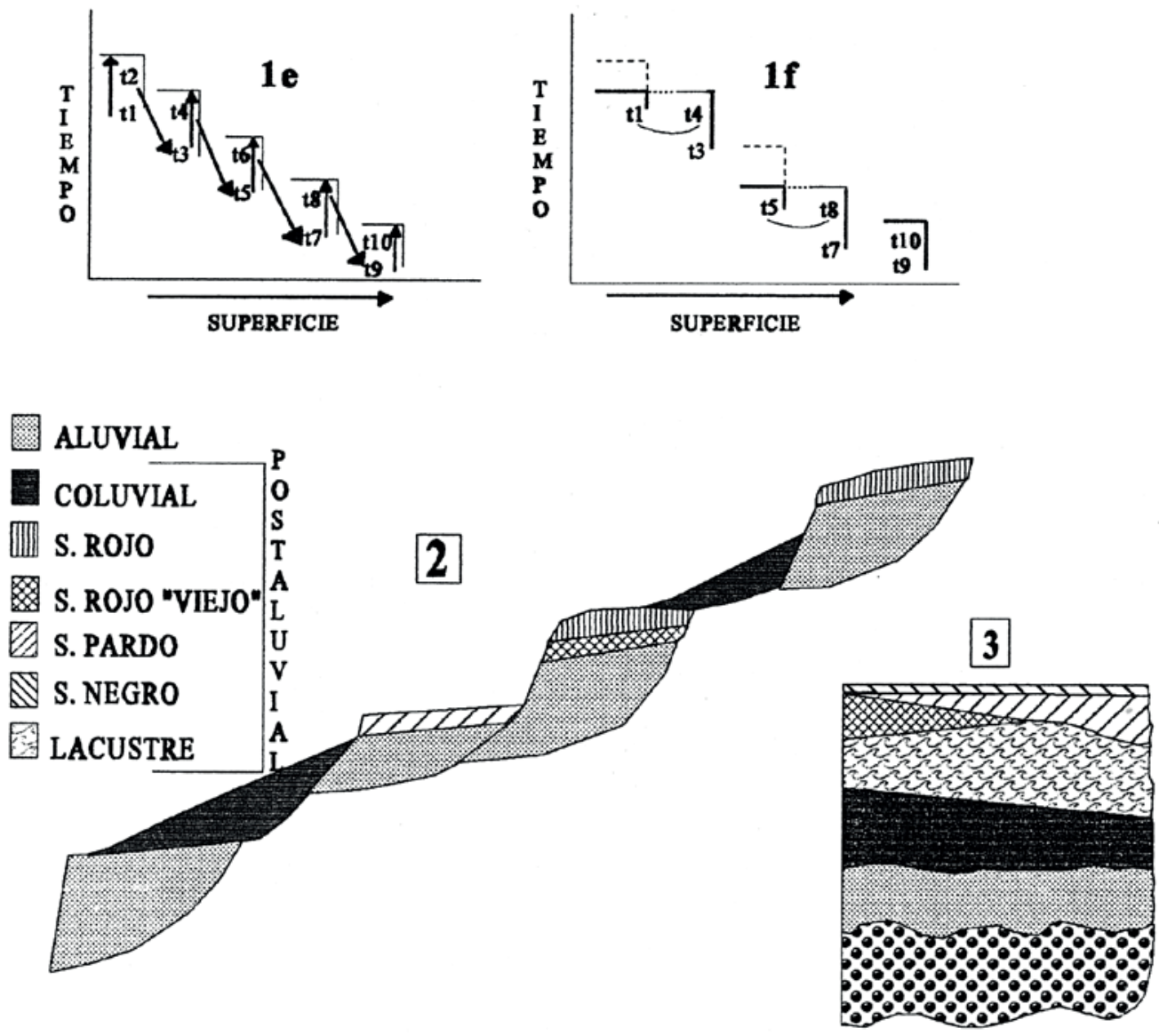

Fig. 2: Interpretación de las terrazas fluviales (definición 1a, formación 1b, estratigrafía 1c, morfología $1 \mathrm{~d}$ y relación cronológica 1e, 1f). Resumen arqueológico interpretativo del tipo de yacimientos y localizaciones en el Paleolítico Inferior y Medio del Bajo Valle del Guadalquivir: 2, general y 3, local. 


\begin{tabular}{|c|c|c|c|c|c|c|c|c|c|c|c|c|c|c|c|c|c|c|}
\hline \multirow[t]{2}{*}{ Terraza } & \multicolumn{2}{|c|}{$\begin{array}{c}\mathrm{T} 5 \\
+139 \mathrm{~m} .\end{array}$} & \multicolumn{2}{|c|}{$\begin{array}{c}\mathrm{T} 6 \\
+110-1.15 \mathrm{~m}\end{array}$} & \multicolumn{2}{|c|}{$\begin{array}{c}\mathrm{T} 7 \\
+95-100 \mathrm{~m}\end{array}$} & \multicolumn{2}{|c|}{$\begin{array}{c}\mathrm{T} 8 \\
+80-85 \mathrm{~m} .\end{array}$} & \multicolumn{2}{|c|}{$\begin{array}{c}\mathrm{T} 9 \\
+73-75 \mathrm{~m} .\end{array}$} & \multicolumn{2}{|c|}{$\begin{array}{c}\mathrm{T} 10 \\
+45-55 \mathrm{~m} .\end{array}$} & \multicolumn{2}{|c|}{$\begin{array}{c}\mathrm{T} 11 \\
+35-40 \mathrm{~m}\end{array}$} & \multicolumn{2}{|c|}{$\begin{array}{c}\mathrm{T} 12 \\
+26-29 \mathrm{~m} .\end{array}$} & \multicolumn{2}{|c|}{ TOTAL } \\
\hline & $\mathrm{N}$ & $\%$ & $\mathrm{~N}$ & $\%$ & $\mathrm{~N}$ & $\%$ & $\mathrm{~N}$ & $\%$ & $\mathrm{~N}$ & $\%$ & $\mathrm{~N}$ & $\%$ & $\mathrm{~N}$ & $\%$ & $\mathrm{~N}$ & $\%$ & $\mathrm{~N}$ & $\%$ \\
\hline Gravas & 52 & 2.2 & 0 & 0.0 & 99 & 4.3 & 71 & 3,1 & 61 & 2.6 & 49 & 2.1 & 881 & 38.0 & 1103 & 47.6 & 2316 & 17.7 \\
\hline Arenas & 0 & 0.0 & 0 & 0.0 & 0 & 0.0 & 0 & 0.0 & 18 & 29.0 & 0 & 0.0 & 7 & 11.3 & 37 & 59.7 & 62 & 0.5 \\
\hline Carbonatos & 9 & 20.0 & 23 & 51.1 & 5 & 11.1 & 8 & 17.8 & 0 & 0.0 & 0 & 0.0 & 0 & 0.0 & 0 & 0.0 & 45 & 0.3 \\
\hline Llanura & 0 & 0.0 & 0 & 0.0 & 272 & 7.8 & 17 & 0.5 & 0 & 0.0 & 312 & 9.0 & 2653 & 76.2 & 227 & 6.5 & 3481 & 26.6 \\
\hline S. Rojo & 0 & 0.0 & 0 & 0.0 & 84 & 87.5 & 12 & 12.5 & 0 & 0.0 & 0 & 0.0 & 0 & 0.0 & 0 & 0.0 & 96 & 0.7 \\
\hline Col. Rojo & 116 & 5.1 & 253 & 11.1 & 1133 & 49.7 & 40 & 1.8 & 467 & 20.5 & 269 & 11.8 & 0 & 0.0 & 0 & 0.0 & 2278 & 17.4 \\
\hline Col. Pardo & 0 & 0.0 & 0 & 0.0 & 27 & 30.7 & 3 & 3.4 & 4 & 4.5 & 45 & 51.1 & 9 & 10.2 & 0 & 0.0 & 88 & 0.7 \\
\hline Arroyos & 0 & 0.0 & 0 & 0.0 & 1411 & 29.9 & 1256 & 26.6 & 896 & 19.0 & 273 & 5.8 & 857 & 18.2 & 20 & 0.4 & 4713 & 36.0 \\
\hline Total & 177 & 1.4 & 276 & 2.1 & 3031 & 23.2 & 1407 & 10.8 & 1446 & 11.1 & 948 & 7.2 & 4407 & 33.7 & 1387 & 10.6 & 13079 & 100 \\
\hline
\end{tabular}

Fig. 3: Relación entre los diferentes niveles de terraza del Bajo Guadalquivir, las formaciones cuaternarias del Pleistoceno correspondientes y la representatividad de las series industriales recuperadas en cada una de ellas. 

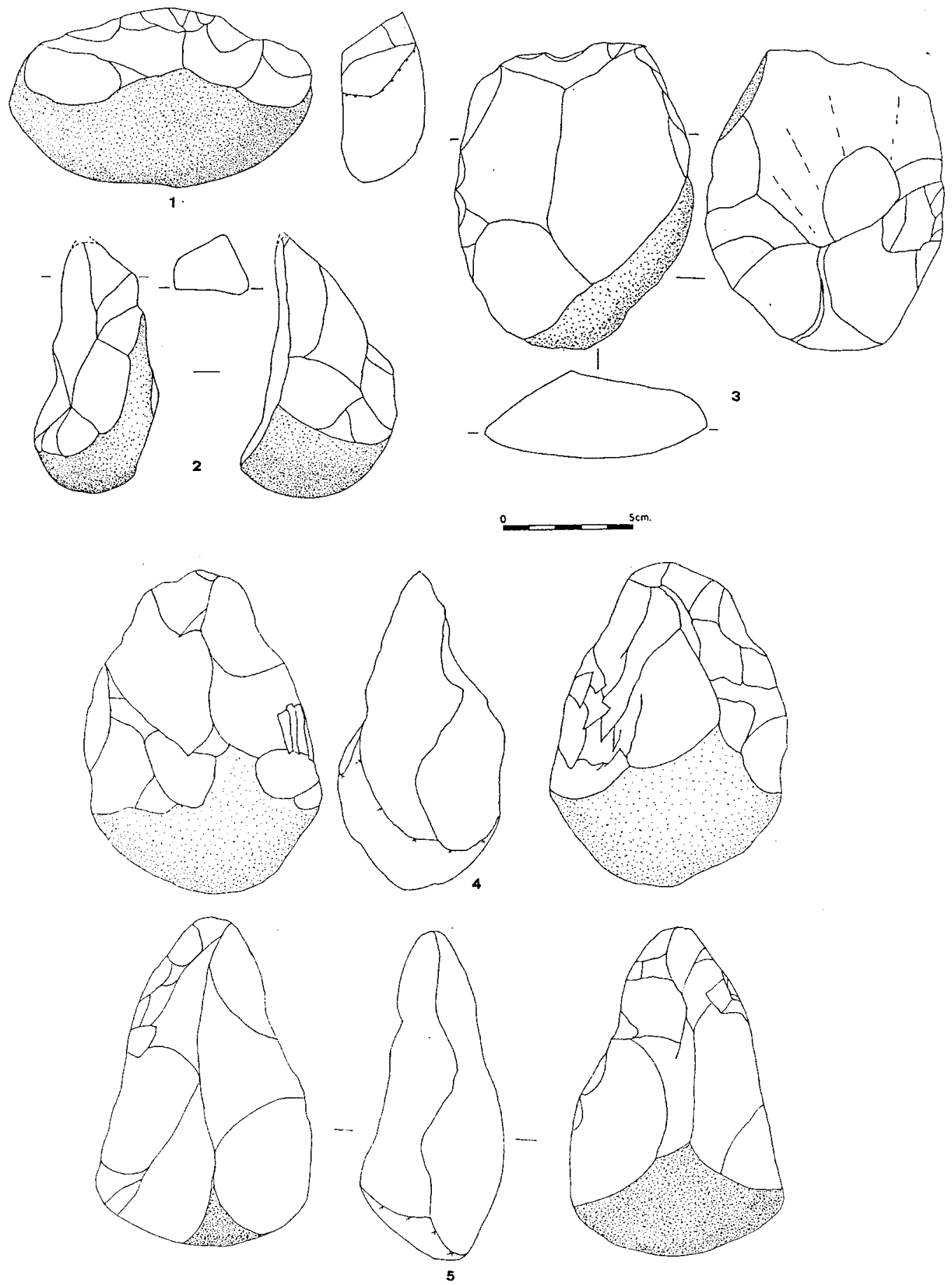

Fig. 4: Industria lítica representativa del Achelense del Bajo Guadalquivir. Huerta de la Reina (coluvión -T6-): canto tallado (1) y triedro (2). La Quintana (coluvión -T9-): hendedor tipo II (3). Buena Esperanza (afluente secundario-T9-): bifaz amigdaloide corto con talón (4). Las Adelfas (afluente secundario-T10-): bifaz lanceolado (5).

ISSN: 1133-4525 ISSN-e: 2255-3924 


\section{Diagrama1}

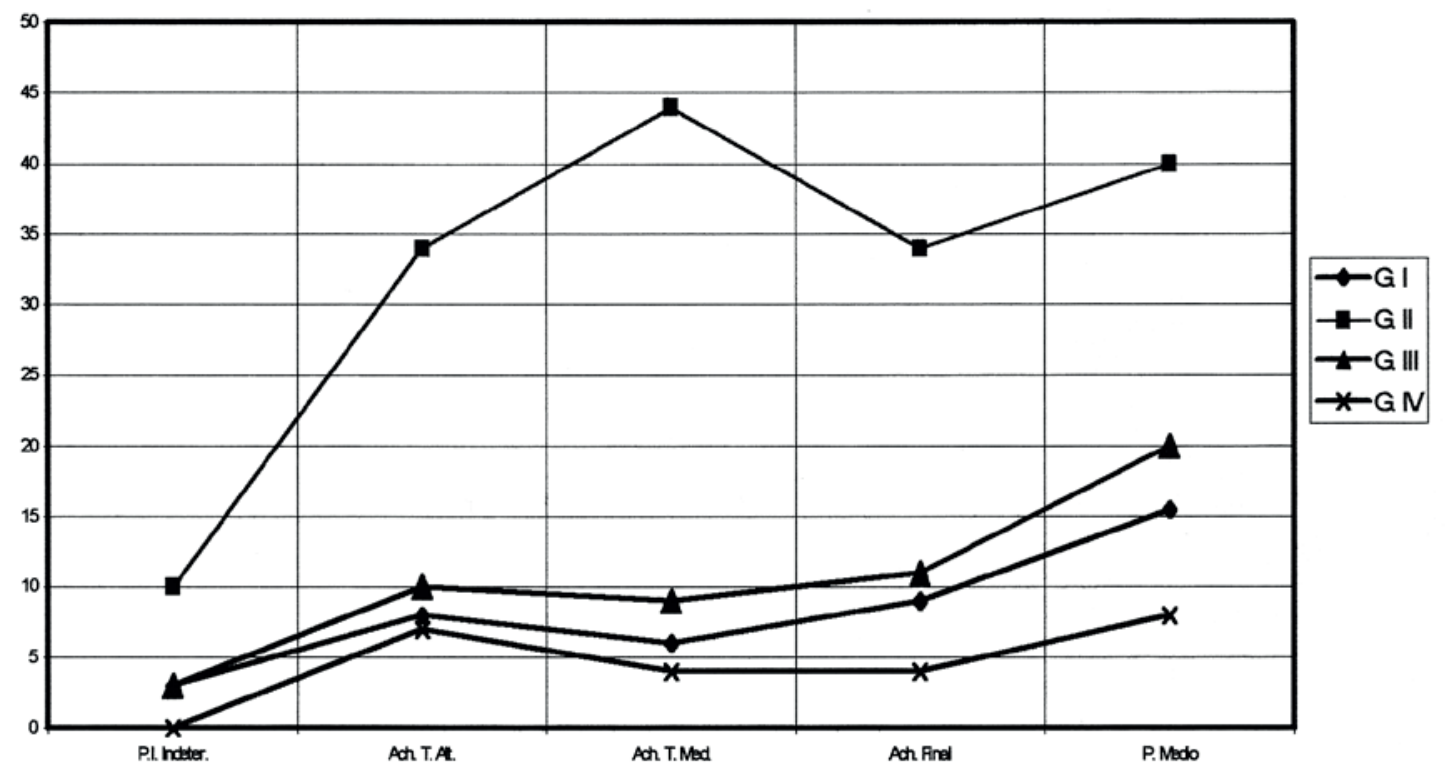

Diagrama 2

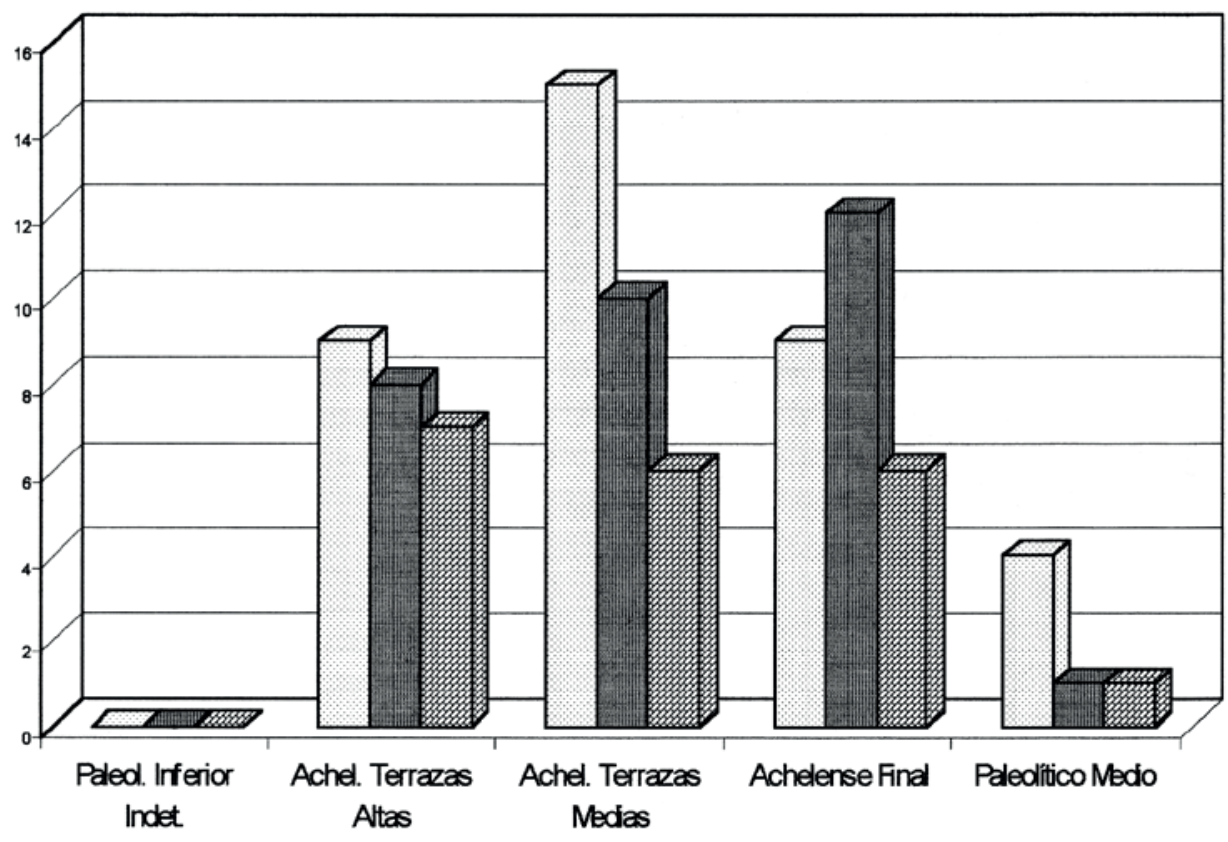

Fig. 5: Evolución de los índices de grupos tipológicos (diagrama 1) y del macroutillaje (diagrama 2) a lo largo del Paleolítico Inferior y Medio del Bajo Guadalquivir. 


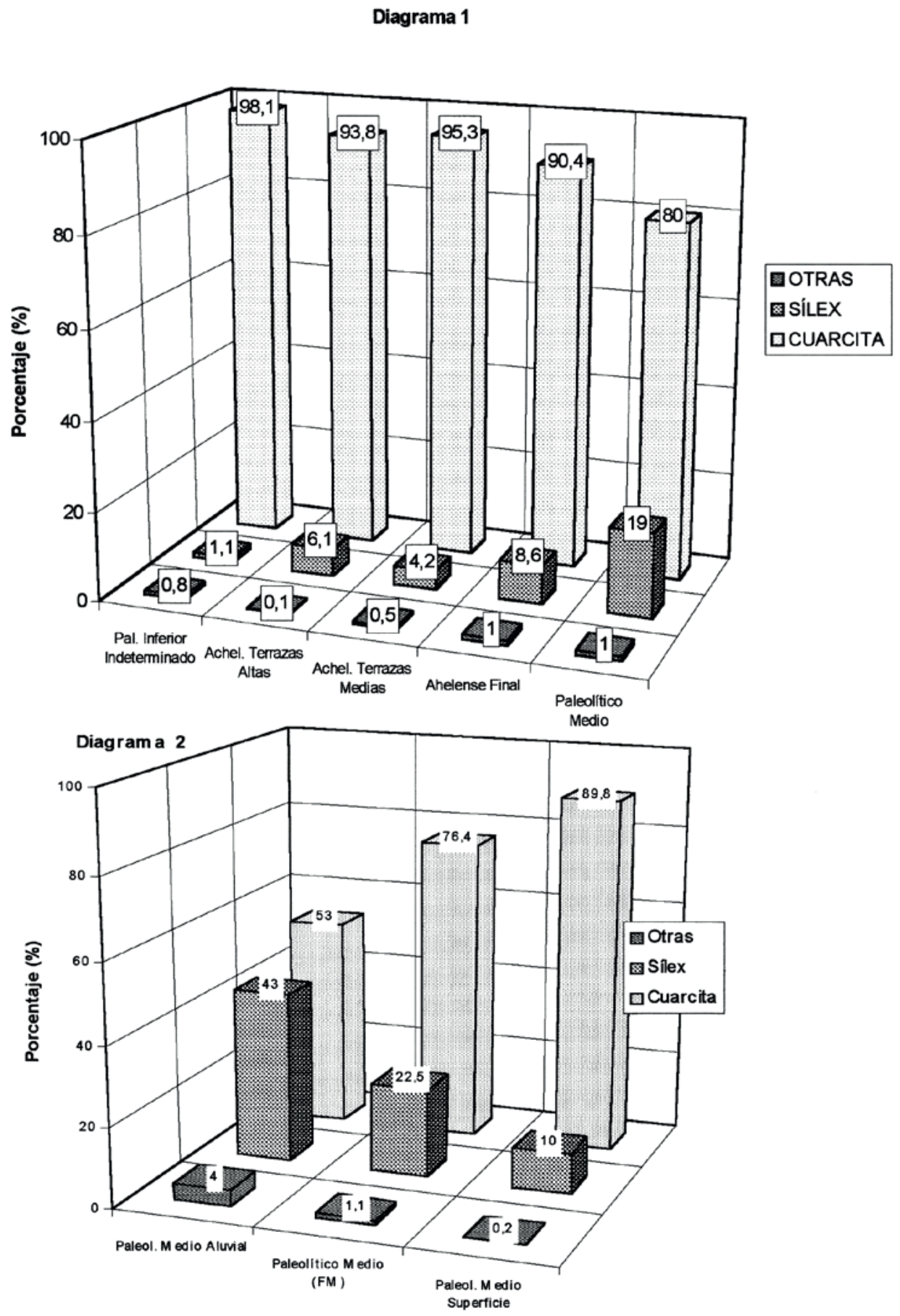

Fig. 6: Utilización de la materia prima durante el Paleolítico Inferior y Medio en el valle del Bajo Guadalquivir (diagrama 1). En el diagrama 2 se refleja la diferencia en el empleo de la materia prima durante el Paleolítico Medio según se trate de yacimientos en depósitos aluviales, en formaciones de finos a techo (Fm: lacustre, palustre, tierras negras, etc.) o en la superficie de las terrazas. 


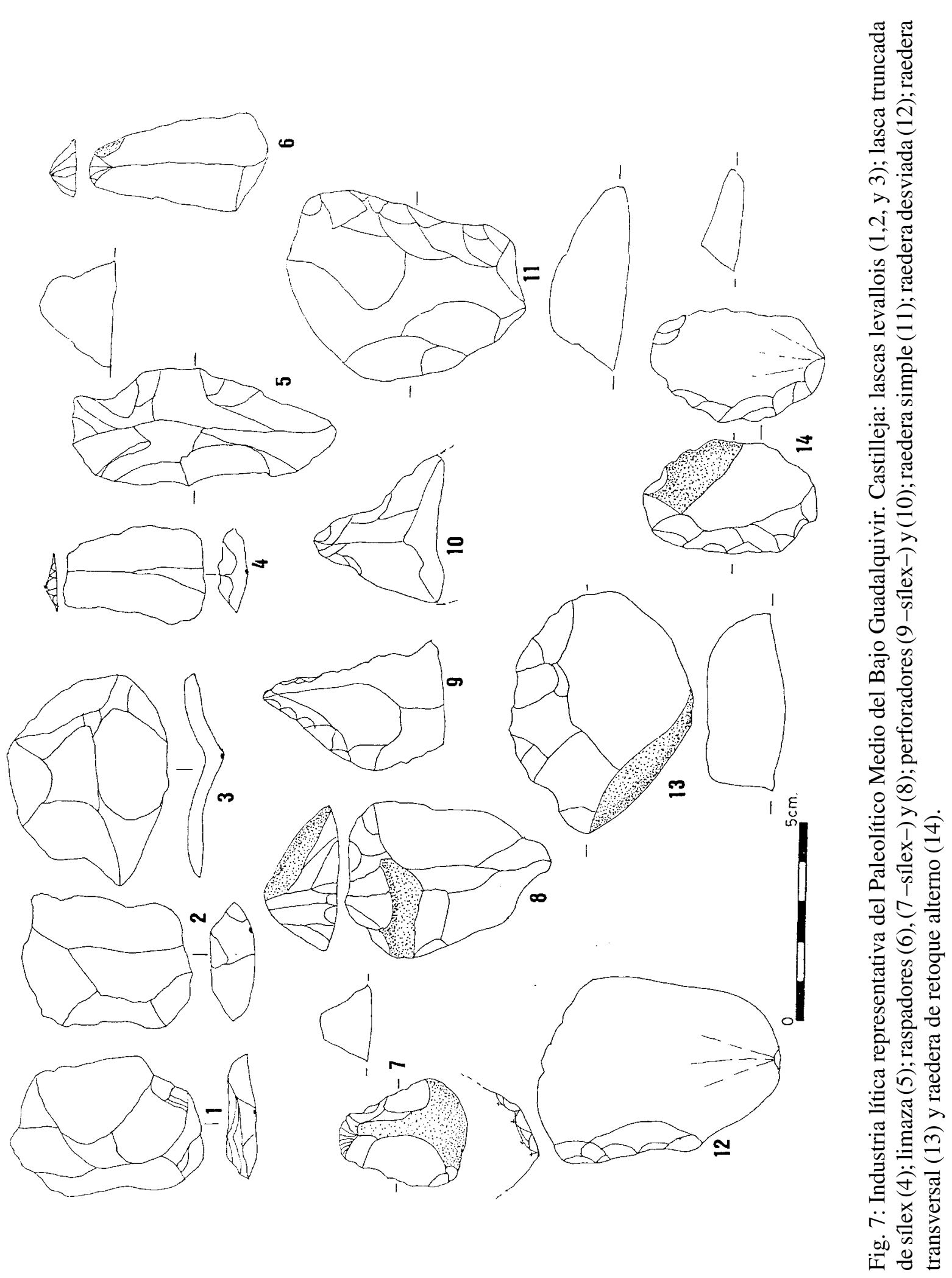




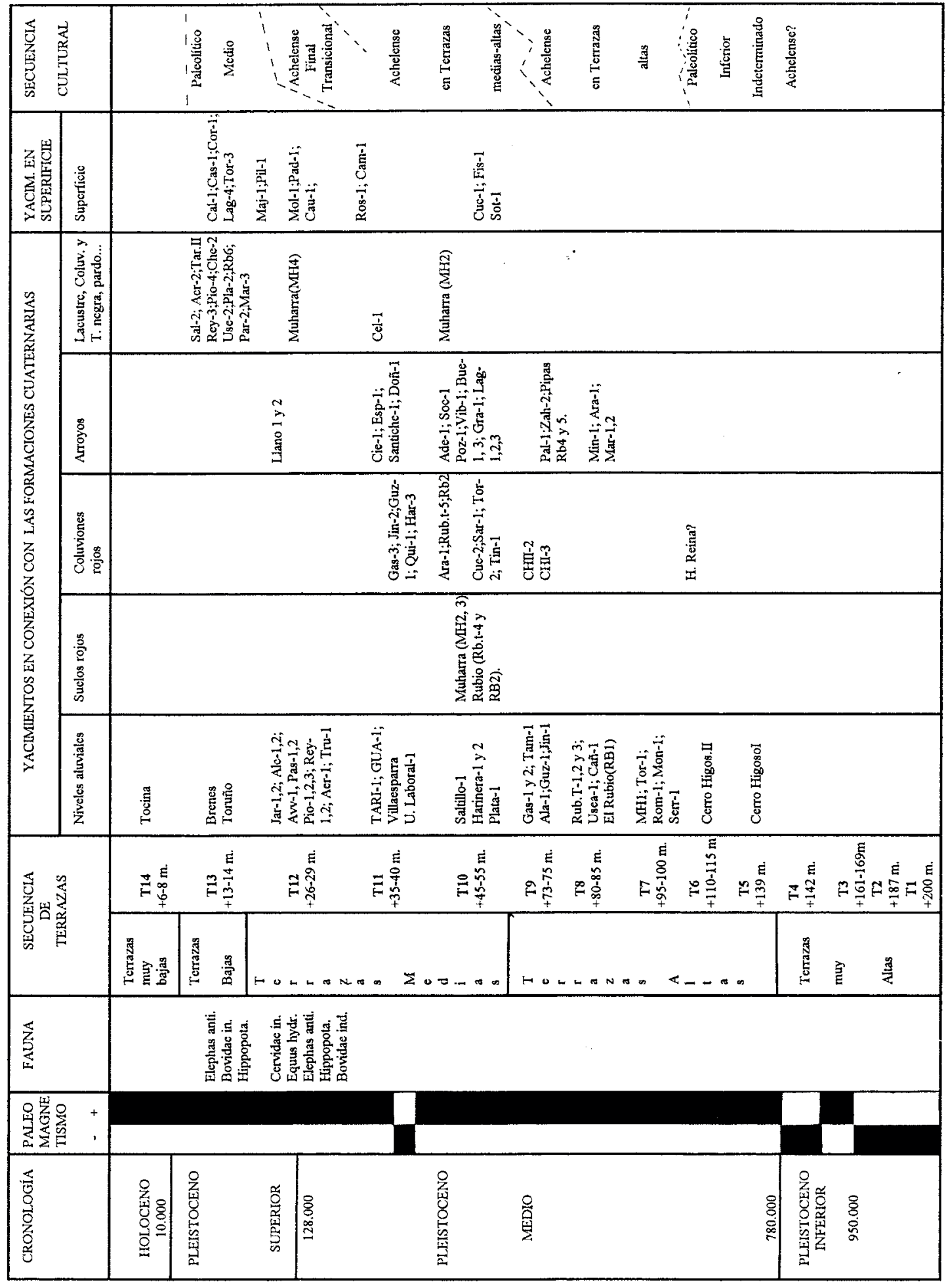

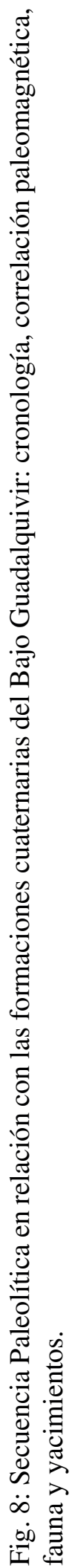

